ARTICLE

\title{
Observation of Weyl fermions in a magnetic non-centrosymmetric crystal
}

Daniel S. Sanchez (10 1,10, Guoqing Chang (1) 1,10, Ilya Belopolski ${ }^{1,10}$, Hong Lu², Jia-Xin Yin (1) ${ }^{1}$, Nasser Alidoust ${ }^{1,3}$,

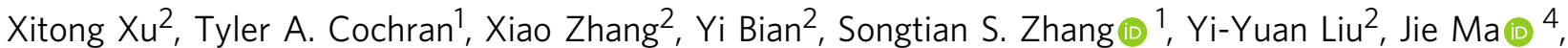
Guang Bian ${ }^{5}$, Hsin Lin (i) ${ }^{6}$, Su-Yang $X u^{1}$, Shuang Jia (i) ${ }^{2,7} \&$ M. Zahid Hasan (iD ${ }^{1,8,9 凶}$

The absence of inversion symmetry in non-centrosymmetric materials has a fundamental role in the emergence of a vast number of fascinating phenomena, like ferroelectricity, second harmonic generation, and Weyl fermions. The removal of time-reversal symmetry in such systems further extends the variety of observable magneto-electric and topological effects. Here we report the striking topological properties in the non-centrosymmetric spin-orbit magnet PrAlGe by combining spectroscopy and transport measurements. By photoemission spectroscopy below the Curie temperature, we observe topological Fermi arcs that correspond to projected topological charges of \pm 1 in the surface Brillouin zone. In the bulk, we observe the linear energy-dispersion of the Weyl fermions. We further observe a large anomalous Hall response in our magneto-transport measurements, which is understood to arise from diverging bulk Berry curvature fields associated with the Weyl band structure. These results establish a novel Weyl semimetal phase in magnetic non-centrosymmetric PrAIGe.

\footnotetext{
${ }^{1}$ Laboratory for Topological Quantum Matter and Advanced Spectroscopy (B7), Department of Physics, Princeton University, Princeton, NJ 08544, USA. ${ }^{2}$ International Center for Quantum Materials, School of Physics, Peking University, Peking, China. ${ }^{3}$ Rigetti Computing, Berkeley, CA 94720, USA. ${ }^{4}$ Key Laboratory of Artificial Structures and Quantum Control, School of Physics and Astronomy, Shanghai Jiao Tong University, Shanghai, China. ${ }^{5}$ Department of Physics and Astronomy, University of Missouri, Columbia, MO, USA. ${ }^{6}$ Institute of Physics, Academia Sinica, Taipei 11529, Taiwan. ${ }^{7}$ Collaborative Innovation Center of Quantum Matter, 100871 Beijing, China. ${ }^{8}$ Princeton Institute for Science and Technology of Materials, Princeton University, Princeton, NJ 08544 USA. ${ }^{9}$ Lawrence Berkeley National Laboratory, Berkeley, CA 94720, USA. ${ }^{10}$ These authors contributed equally: Daniel S. Sanchez, Guoqing Chang, Ilya Belopolski. ${ }^{凶}$ email: mzhasan@princeton.edu
} 
$\mathrm{D}$ evelopment in the search for materials with topological electronic properties has rapidly progressed in the past decade. With a refined understanding of the role symmetries have on the electron wavefunctions Berry curvature, the experimental study of new and exotic quantum phenomena has now become widely accessible ${ }^{1-18}$. A well-recognized example is the breaking of time-reversal symmetry in magnetic materials, which may result in producing Berry curvature fields that generate an intrinsic anomalous Hall response. Along similar lines, the breaking of inversion symmetry in noncentrosymmetric materials is understood to be essential for fostering new quantum phenomena, such as non-local gyrotropic effects $^{19}$, quantum nonlinear Hall effects ${ }^{20}$, photogalvanic effects $^{21}$, accidental two-fold band degeneracies (Weyl fermions) that are protected by a quantized non-zero integer Chern number (chiral charge) ${ }^{22-25}$, and anomalous transport ${ }^{26}$.

In this study, we observe that magnetic non-centrosymmetric PrAlGe ${ }^{14}$ hosts the emergent topological properties of Weyl fermions by photoemission-based spectroscopy and magnetotransport. In contrast to previous works on magnetic Weyl semimetal candidates $\mathrm{Mn}_{3} \mathrm{Sn}^{27,28}$ and $\mathrm{Co}_{3} \mathrm{Sn}_{2} \mathrm{~S}_{2}{ }^{29-31}$ (both centrosymmetric), PrAlGe is calculated to exhibit Weyl fermions in proximity to the Fermi level, making it more suitable for experimentally probing its Berry curvature properties and exploring the connection between photoemission-based band structure and transport. In addition, because PrAlGe lacks both inversion and time-reversal symmetry it can uniquely induce quantum spin currents without a concomitant charge current ${ }^{14,15}$. Motivating future studies on PrAlGe, we experimentally resolve the key topological properties of Weyl fermions by relying predominately on our measurements ${ }^{11,32}$.

\section{Results}

Magnetic and electronic properties. PrAlGe crystallizes in a body-centered tetragonal Bravais lattice with space group $I 4_{1} m d$ (No. 109). The basis consists of two Pr, two $\mathrm{Al}$ and two Ge atoms, Fig. 1a inset. Along the (001) direction, each atomic layer is comprised of one element, and the layer is shifted relative to the one below by half a lattice constant in either the $x$ or $y$ direction. Single crystal X-ray diffraction suggests that our samples possess the correct lattice structure and lack inversion symmetry (Supplementary Table 1). Measurements of magnetic susceptibility as a function of temperature were fitted to the inverse Curie-Weiss law. The obtained positive Weiss constant indicates the presence of ferromagnetic interactions, Fig. 1a. A direct measurement, to be discussed below, shows that PrAlGe is ferromagnetic with Curie temperature $T_{\mathrm{C}}=16 \mathrm{~K}$. The ferromagnetic ground state arises from the spin-polarized $f$-electron states that are locally coupled and aligned along the $c$ axis, rendering the conduction electron bands near the Fermi level spin-polarized. This is reflected in our ab initio band structure calculations without spinorbit coupling (SOC), in which it also shows that PrAlGe has a semi-metallic profile (top panel: Fig. 1b). The inclusion of SOC a

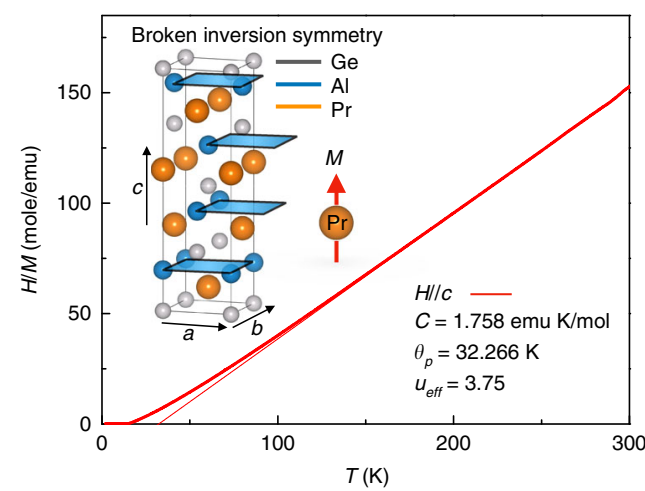

b

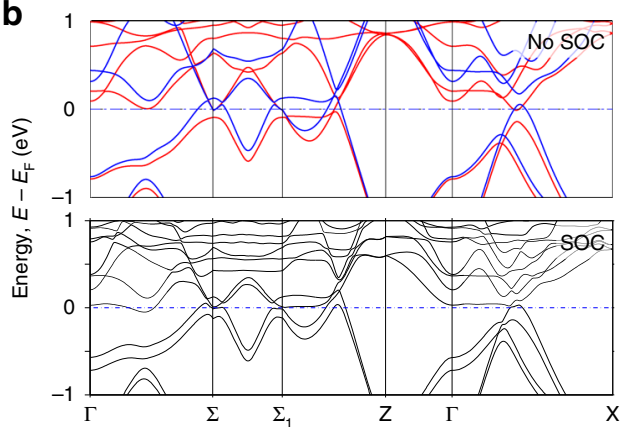

C

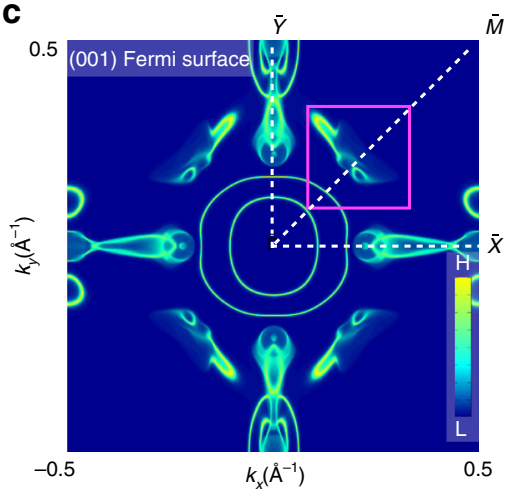

d

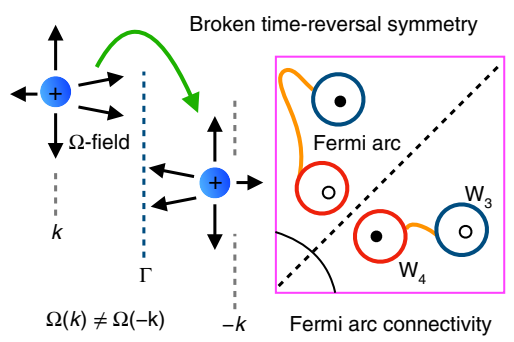

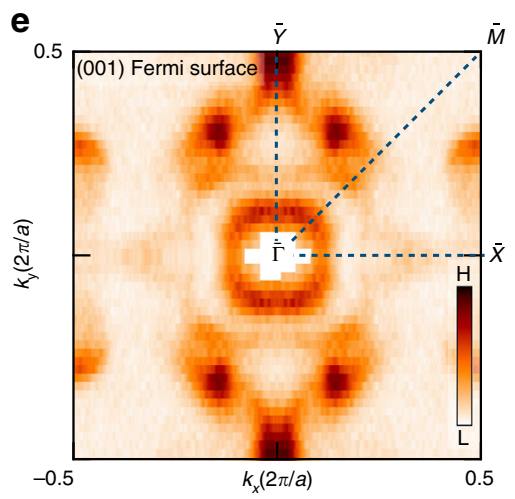

f

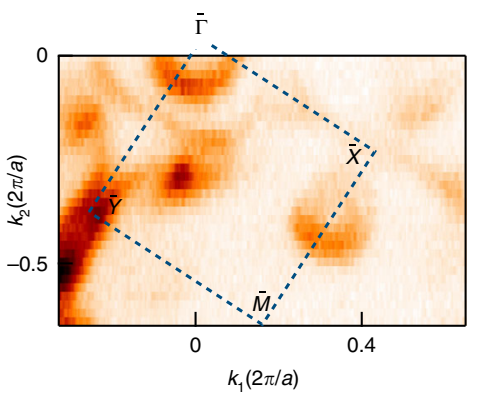

Fig. 1 Lattice and electronic structure of non-centrosymmetric spin-orbit magnet PrAIGe. a Inverse magnetic susceptibility as a function of temperature (thick line) with fit to a Curie-Weiss law (thin line). Curie temperature $T_{\mathrm{C}}=16 \mathrm{~K}$ was measured. Inset: crystal structure of PrAlGe in space group $14{ }_{1} m d$ (No. 109). The square stacking pattern results in broken inversion symmetry. b Ab initio calculated bulk band structure of PrAIGe without (top panel) and with (bottom panel) spin-orbit coupling. The spin-up and spin-down states are shown in red and blue, respectively. c Ab initio calculated Fermi surface for the (001) surface. White dashed box: first quadrant of the surface BZ. d Left panel: breaking time-reversal symmetry allows the Weyl fermions (+), represented as sources of Berry curvature $\boldsymbol{\Omega}$ fields, to be shifted in the crystal momentum space so they are no longer appear pairwise at $\pm k$. Right panel: Fermi arc connectivity (orange lines) schematic for the projected Weyl fermions (black and white circles), corresponding to the magenta box in c. A pair of projected $W_{3}$ and $W_{4}$ Weyl fermions on each side of the $\bar{\Gamma}-\bar{M}$ line. The predicted configuration of projected Weyl fermions manifestly breaks timereversal symmetry. e Symmetrized and $\mathbf{f}$ un-symmetrized Fermi surface obtained by low-energy ARPES. 

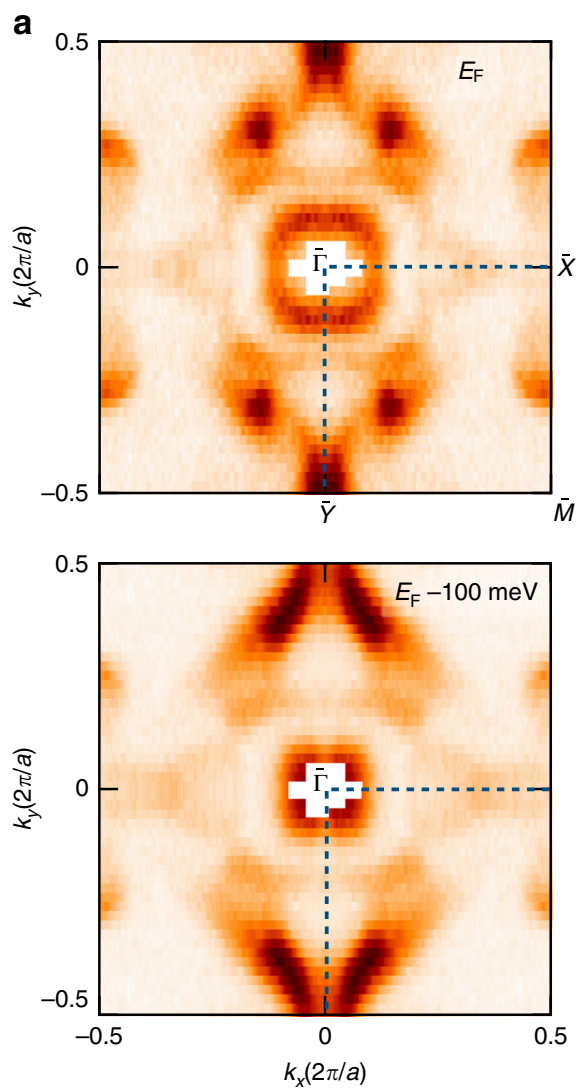
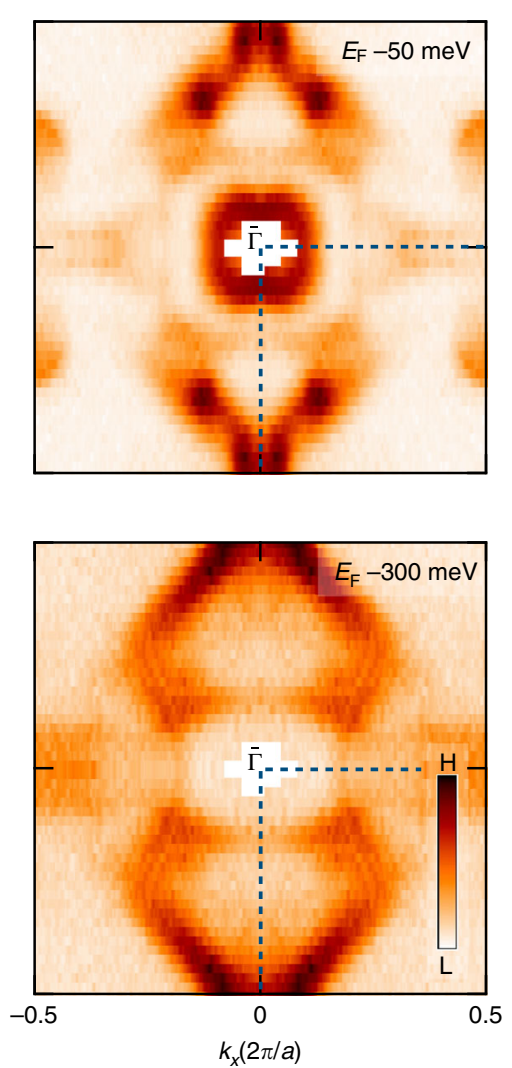
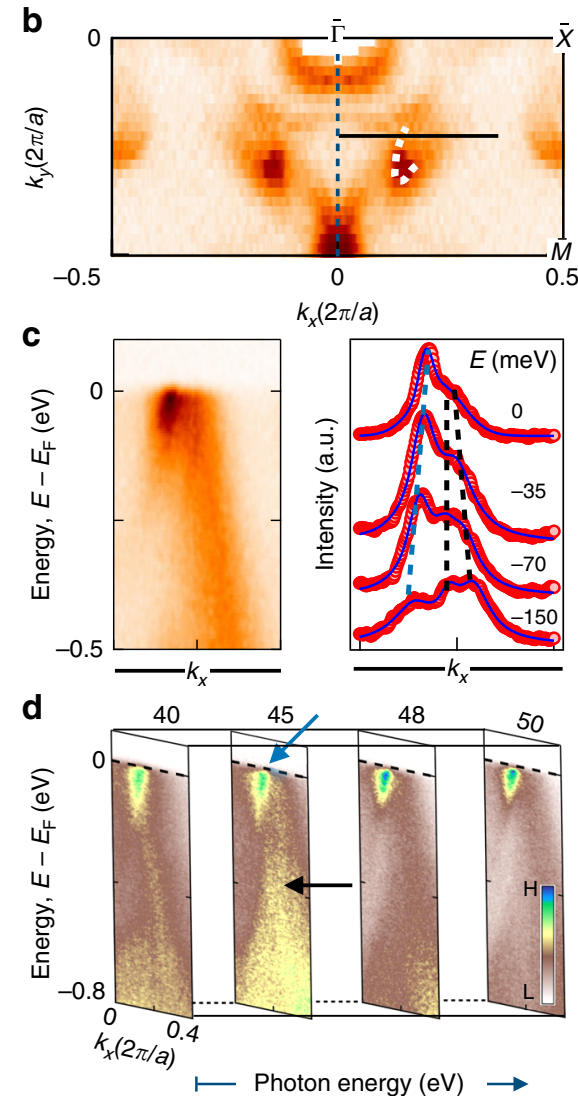

Fig. 2 Fermiology and topology of the (001) surface electronic structure in PrAIGe. a Low-energy ARPES-measured Fermi surface and constant binding energy contours obtained with incident photon energies of $50 \mathrm{eV}$ at $T \approx 11 \mathrm{~K}$. Blue dashed line: one quadrant of the surface Brillouin zone. $\mathbf{b}$ ARPESmeasured Fermi surface with guides to the eye (white dashed line) tracking the " $U$ " shaped candidate topological Fermi arc state. c Left: energymomentum cut and, right: MDCs fitted at different binding energies with Lorentzian functions to track the candidate arc (blue dashed line) and bulk states (black dashed lines). The corresponding path is shown in b. d Photon-energy dependent ARPES along the horizontal line in $\mathbf{b}$. Negligible $k_{z}$ dispersion is observed for the candidate " $U$ " shaped topological Fermi arc (blue arrow). Strong photon-energy dependence is observed for other states nearby (black arrow), suggesting that they are bulk states.

interactions couples the spin-up and spin-down states and slightly perturbs the electronic bands (bottom panel: Fig. 1b). The absence of inversion and time-reversal symmetry both contribute to band-splitting at generic crystal momenta. Kramers degeneracy splitting is linked to magnetism in the crystal. Of the Weyl fermions predicted in ferromagnetic PrAlGe ${ }^{14}$, two groups (labeled $\mathrm{W}_{3}$ and $\mathrm{W}_{4}$ ) are within $\pm 20 \mathrm{meV}$ of the Fermi level. The ab initio calculated Fermi surface for ferromagnetic PrAlGe predicts the presence of topological Fermi arcs in each quadrant, with an asymmetry across the $\bar{\Gamma}-\bar{M}$ surface high-symmetry line, Fig. 1c, $\mathrm{d}$. The Fermi arc asymmetry is connected to the absence of both inversion and time-reversal symmetry in PrAlGe (Supplementary Figs. 1-3).

VUV-ARPES study of the (001) surface electronic structure. Motivated by our ab initio calculations and transport measurement observing ferromagnetism, we use angle-resolved photoemission spectroscopy (ARPES) measurements at low-photon energies (VUV-ARPES) to map the band structure of PrAlGe on the (001) surface at temperature $T=11 \mathrm{~K}$, below $T_{\mathrm{C}}$. We observe that the ab initio calculation is qualitatively consistent with the measured Fermi surface, Fig. 1e, f. On constant-energy contours of varying binding energy, we observe the following dominant features, Fig. 2a: two concentric closed contours around the $\bar{\Gamma}$ point, a distinct " $U$ " shaped state (marked by a guide to the eye, Fig. 2b), and additional surface states near the $\bar{Y}$ of the surface
BZ. The inner closed concentric contour shrinks with deeper binding energy, showing a clear electron-like behavior. To better understand the nature of the " $U$ " state and the spectral intensity in its vicinity, we study an energy-momentum cut through this state, Fig. 2c. We plot the Lorentzian-fitted momentum distribution curves (MDCs) at different binding energies and find that the "U" state disperses towards the Fermi level while a nearby band approaches $E_{\mathrm{F}}$ and then turns back toward deeper binding energies. We also find that the " $U$ " state exhibits negligible photon energy dependence, suggesting that it is a surface state (Fig. 2d, Supplementary Fig. 4). A comparison with the ab initio calculated Fermi surface further suggests that the " $U$ " state corresponds to the predicted Fermi arc connecting $\mathrm{W}_{3}$ and $\mathrm{W}_{4}$. The surface state nature of the " $U$ " state and its correspondence with calculation suggests that the ARPES-measured state is a topological Fermi arc.

Topological Fermi arcs and projected chiral charges. To further explore the Fermi arc candidate we search for direct spectroscopic signatures of chiral charges in PrAlGe, taking advantage of the bulk-boundary correspondence between bulk Weyl fermions and surface Fermi $\operatorname{arcs}^{11,32}$. We study chiral edge modes along straight and loop energy-momentum cuts (Fig. 3a, b), and present a two-dimensional curvature plot of the measured Fermi surface to further highlight the momentum space trajectory of the Fermi arc candidate, Fig. 3c. A horizontal momentum cut at 
a

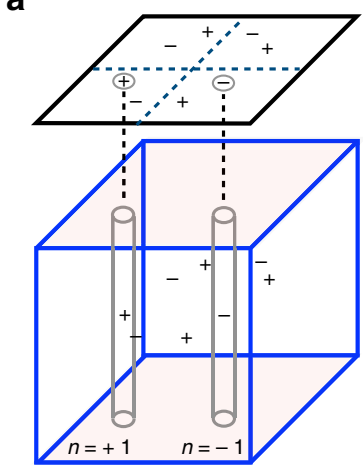

b

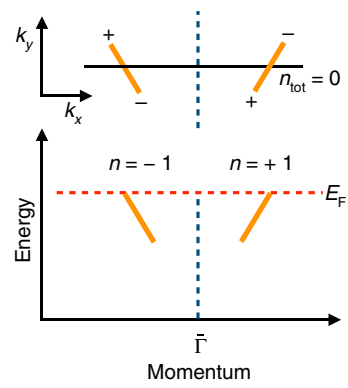

c

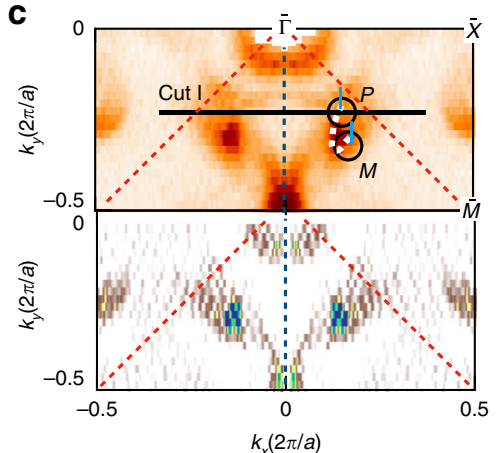

f

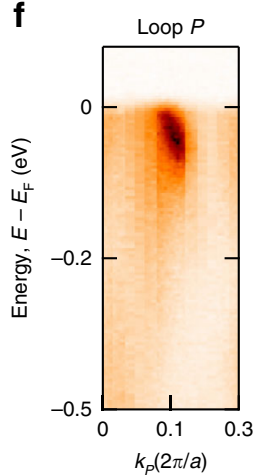

g d
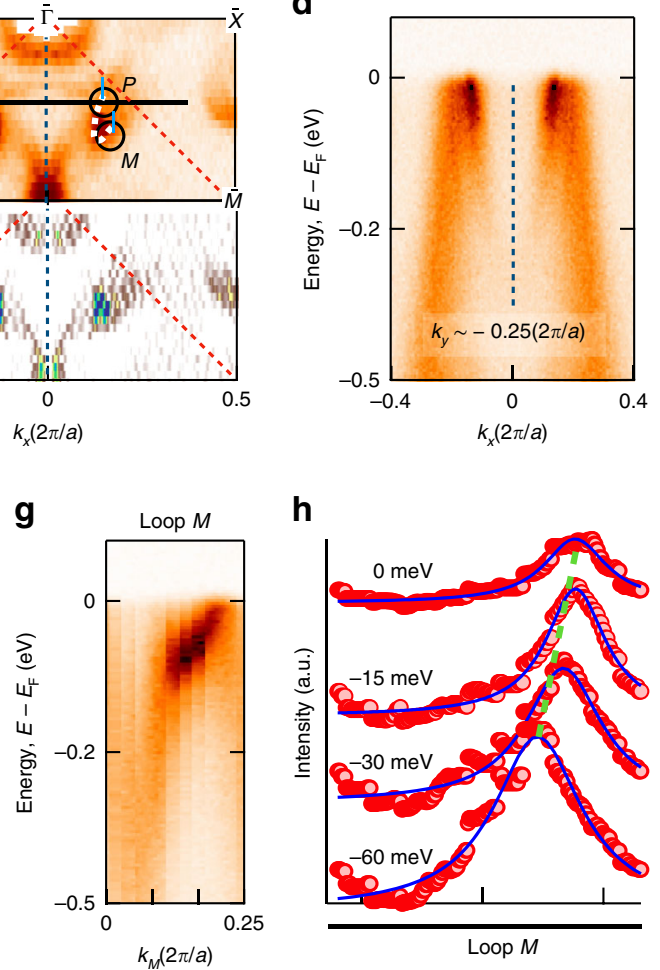

h

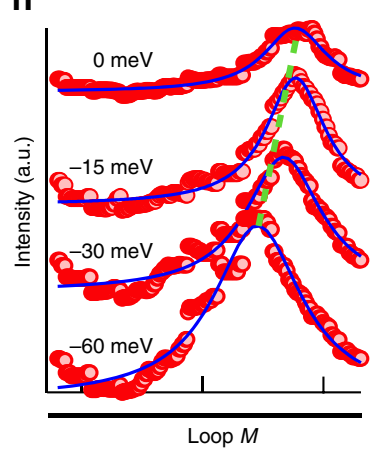

e

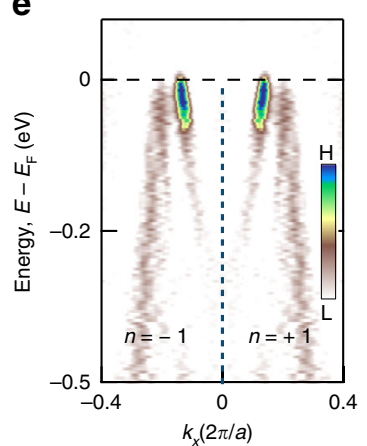

i

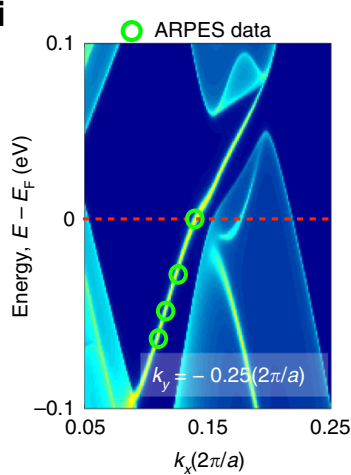

Fig. 3 Observation of topological Fermi arcs and chiral charges in PrAIGe. a Bulk and surface Brillouin zone (BZ) with the Weyl fermions ( \pm ) and manifolds with Chern number $n^{32}$. A closed loop enclosing the projected chiral charge in the surface corresponds to a cylinder in the bulk enclosing the Weyl fermion. b Top: topological Fermi arcs (orange) connecting the projected Weyl fermions carrying chiral charge \pm 1 . Bottom: a cut across two arcs (along the black line in top panel) with chiral edge modes (orange lines). c Top panel: Fermi surface obtained by ARPES at $T \approx 11 \mathrm{~K}$. Loop cuts of interest $(P$ and $M)$ are illustrated with black loops with the starting/end points marked by the vertical blue line. Bottom panel: 2D curvature plot of the above Fermi surface. d Measured band dispersion along horizontal Cut I. Blue dashed line: indicates the mirror symmetry. e Second-derivative plot of $\mathbf{d}$. $\mathbf{f}$ ARPESmeasured band dispersion along the loop P. Loop $P$ encloses the termination point of the measured Fermi arc and shows a single left-moving chiral mode, corresponding to an enclosed Chern number $n=-1$. $\mathbf{g}$ Band dispersion along loop $M$. The observed right-moving chiral mode shows an enclosed Chern number $n=+1$. $\mathbf{h}$ Stack of MDCs along loop $M$ at different binding energies, with Lorentzian fits. Green dashed line: guide to the eye tracking the peaks. i Calculated energy dispersion along $k_{y}=-0.25(2 \pi / a)$ with the result from our ARPES spectra overlaid (open green circles).

$k_{y} \approx-0.25(2 \pi / a)$ passes through a pair of " $U$ " states, Fig. $3 \mathrm{c}, \mathrm{d}$. We observe signatures of a left-moving and right-moving mode related by mirror symmetry. A second derivative plot of the dispersion map further confirms the observed modes and suggests additional neighboring bulk bands which approach $E_{\mathrm{F}}$ and then turn back towards deeper binding energies, Fig. 3e. A comparison of this spectrum with the locations of the predicted Weyl fermions suggests that we can interpret the left- and rightmoving modes as two chiral edge modes, associated with Chern number $n= \pm 1$, Fig. $3 \mathrm{~b}$. In this way, the momentum cut is associated with a $2 \mathrm{D}$ momentum-space slice carrying Chern number $n_{\text {tot }}=0$, since $n_{1}=-1$ and $n_{\mathrm{r}}=+1$. This again suggests that the left- and right-moving modes giving rise to two mirror partnered " $U$ " states are topological Fermi arcs.

To provide further evidence of chiral charges in PrAlGe, we next perform an analysis of edge modes along closed loops in the surface BZ (black dashed circles labelled $P$ and $M$, Fig. 3c). By counting chiral edge modes on these circular paths, we search for evidence of $n_{\text {tot }} \neq 0$. Unrolling the energy-momentum dispersion for loop $P$, we observe one left-moving chiral mode that is dispersing towards $E_{\mathrm{F}}$, Fig. $3 \mathrm{f}$. This result unambiguously shows chiral charge -1 on the associated bulk manifold. Analogously, along loop $M$ we observe a right-moving chiral mode dispersing towards $E_{\mathrm{F}}$, Fig. $3 \mathrm{~g}$. By Lorentzian fitting of the MDCs along loop $M$ at varying binding energies, we again observe a right-moving mode, Fig. $3 \mathrm{~h}$, suggesting that the corresponding bulk manifold encloses chiral charge +1 . As an additional check, the ab initio band dispersion calculation along $k_{y}=-0.25(2 \pi / a)$ shows a right-moving chiral mode dispersing toward $E_{\mathrm{F}}$, Fig. 3i. An overlay of the Lorentzian fits of the chiral mode on the calculated band dispersion shows a match between our results (Supplementary Fig. 5). In this way, our low-energy ARPES spectra directly resolve topological Fermi arcs and demonstrate chiral charges in PrAlGe through the bulk-boundary correspondence ${ }^{11,32}$. Further, the observed Fermi arc asymmetry across the $\bar{\Gamma}-\bar{M}$ is consistent with our ab initio calculations for PrAlGe (Supplementary Fig. 2).

Bulk Weyl cone dispersion. Next, we provide a comparison between the experimental bulk band structure obtained by ARPES at soft X-ray energies (SX-ARPES) and our ab initio calculations (Fig. 4). To demonstrate the linear dispersion of the bulk Weyl cones, our analysis looks at the energy-dispersion maps along various horizontal and vertical paths that intersect the $\mathrm{ab}$ initio calculated positions of the Weyl fermions on the $k_{z}=0$ plane, Fig. 4a. The large probing depth provided by SX-ARPES allows for a comparison between the ab initio calculated (Fig. 4b) and experimentally measured (Fig. 4c) bulk Fermi surface on the $k_{z}=0$ plane. Both are qualitatively consistent and show an absence of asymmetry across the $\bar{\Gamma}-\bar{M}$ line. Along vertical cut 1 (green line: Fig. 4d) and horizontal cut 2 (blue line: Fig. 4e), we 
a

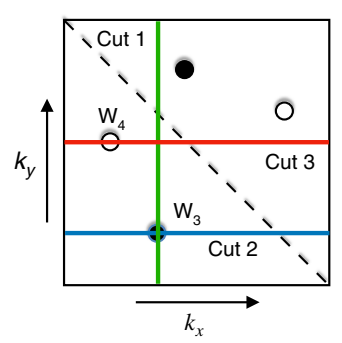

b
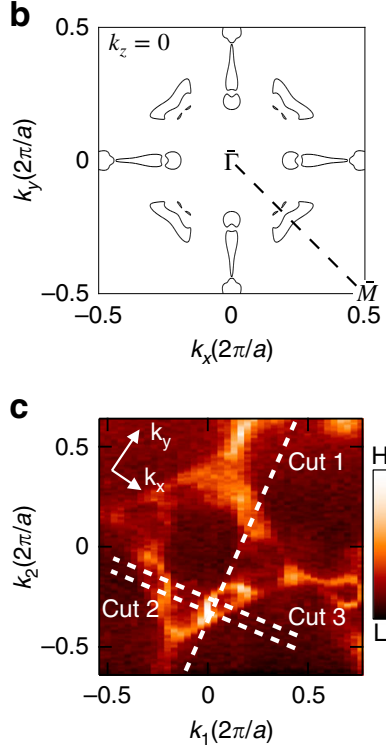

d

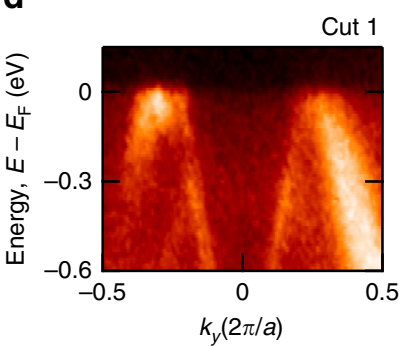

e

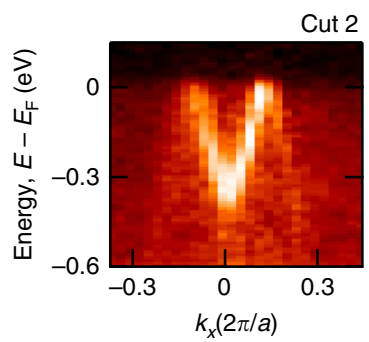

f

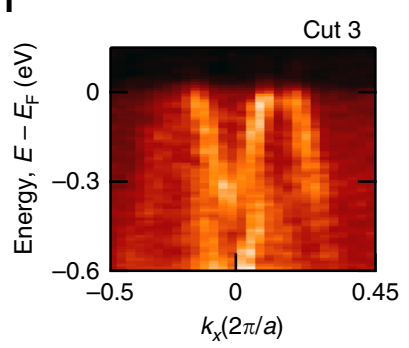

g

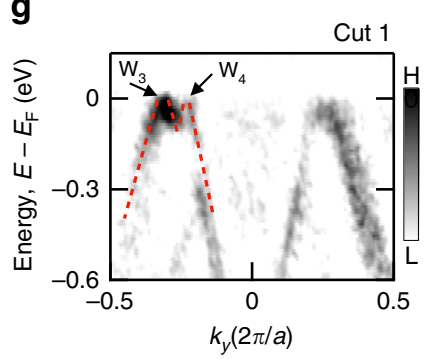

h

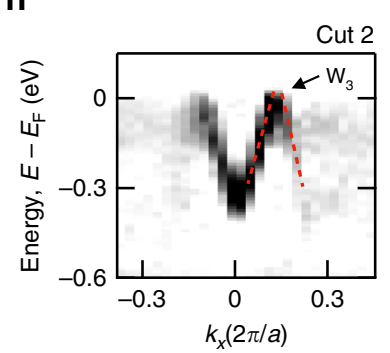

i

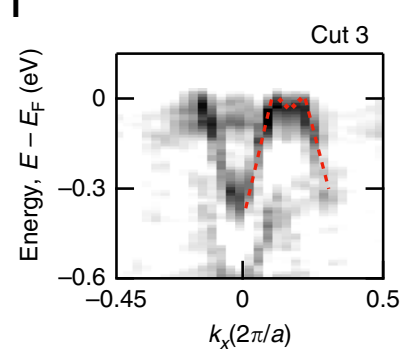

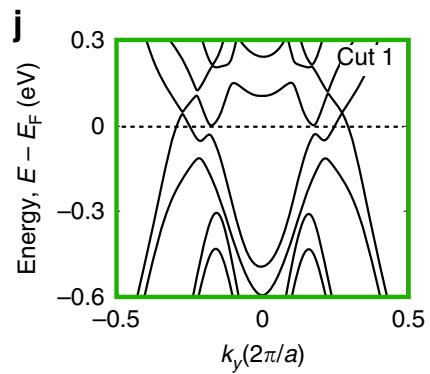

k

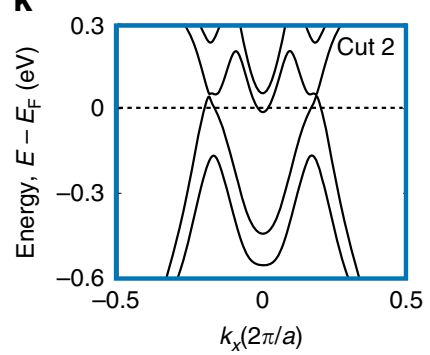

|

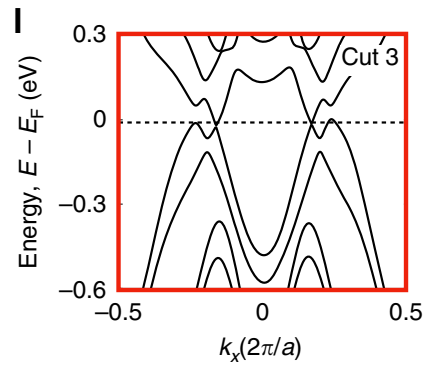

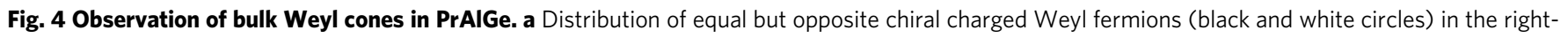

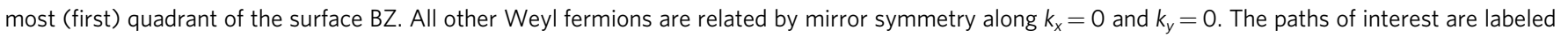
as Cut 1 (green vertical line), Cut 2 (blue horizontal line), and Cut 3 (red horizontal line). b Ab initio calculated bulk Fermi surface ( $k_{x}, k_{y}$ ) at $k_{z}=0$.

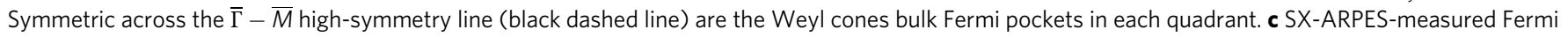

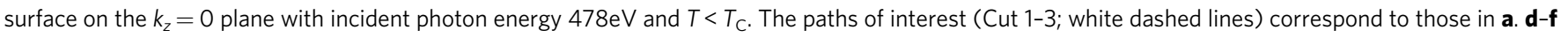

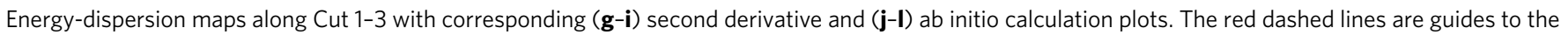
eye for the Weyl cones.

observe the linear dispersion of the $\mathrm{W}_{3}$ and $\mathrm{W}_{4}$ Weyl cones. Horizontal cut 3 (red line: Fig. 4f) further confirmes the linear dispersion of the $\mathrm{W}_{4}$ Weyl fermion. Second derivative plots of Cuts 1-3 with guides to the eye for the Weyl cones further illustrate their linear dispersion (Fig. $4 \mathrm{~g}-\mathrm{i}$ ). Within the resolution of our measurements, the $\mathrm{W}_{3}$ and $\mathrm{W}_{4}$ Weyl fermions are located on the $k_{z}=0$ plane at $(0.15,-0.32) 2 \pi / a$ and $(0.13,-0.22) 2 \pi / a$, respectively. Due to our SX-ARPES measurement temperature being comparable to the Curie temperature of our PrAlGe samples, and limited SX-ARPES energy resolution or spectral linewidth, our data did not clearly resolve the Zeeman splitting, suggesting that further experimental work is needed. Howbeit, the qualitative agreement between our SX-ARPES measurements (Fig. 4d-i) and ab initio calculations (Fig. 4j-l) suggests the observation of bulk Weyl cones in PrAlGe.

Anomalous Hall transport. Having experimentally demonstrated topological Fermi arcs and bulk Weyl cones in PrAlGe, we next investigate additional phenomena mediated by Berry curvature using magneto-transport (Supplementary Fig. 6). We study the magnetization $M$ as a function of magnetic induction $\mu_{0} H$ (Fig. 5a) and observe that PrAlGe is a soft ferromagnet with an easy axis along the $c$-direction. Furthermore, we find that the transverse resistivity $\rho_{y x}$ exhibits an anomalous Hall effect, described by $\rho_{y x}=R_{\mathrm{H}} B+\mu_{0} R_{\mathrm{S}} M$, where $R_{\mathrm{H}}$ is the ordinary (Lorentz-force) Hall coefficient and $R_{\mathrm{S}}$ is the anomalous Hall coefficient $^{33}$. As shown in the inset of Fig. $5 b, R_{\mathrm{S}}$ (zero for high $T$ ) grows rapidly toward large values while the small value for $R_{\mathrm{H}}$ (almost invariant for different $T$ ) decreases very quickly below $T_{\mathrm{C}}$. The observed behavior for $R_{\mathrm{H}}$ and $R_{\mathrm{S}}$ suggests that the anomalous Hall effect arises near $T_{\mathrm{C}}$. The measured $R_{\mathrm{S}}$ coefficient reaches a saturation value of about $1.5 \mu \Omega \mathrm{cm} \mathrm{T}^{-1}$ at $2 \mathrm{~K}$, where it dominates the response $\mathrm{e}^{34,35}$. To investigate the origin of the observed behavior for the anomalous Hall effect, we plotted the anomalous Hall contribution $\rho_{y x}^{A}$ as a function of carrier concentration $p=1$ / $\mathrm{e} R_{\mathrm{H}}$, where $\mathrm{e}$ is the charge of an electron. The result shows clustered values in the vicinity of $1 \mu \Omega \mathrm{cm}$ for different samples, see Fig. $5 c$ inset. To better understand the origin of $\rho_{y x}^{\mathrm{A}}$, we calculated the Berry curvature contribution to the anomalous Hall conductivity, the so-called intrinsic anomalous Hall conductivity, $\sigma_{y x}^{A_{\text {int }}}$ as a function of carrier concentration, Fig. $5 \mathrm{c}$. The calculation predicts a roughly carrier concentration-independent value of $\sim 600 \Omega^{-1} \mathrm{~cm}^{-1}$ with carrier densities from $p=0.9$ to $1.7 \times$ $10^{21} \mathrm{~cm}^{-3}$. This corresponds to an intrinsic contribution to the anomalous Hall resistivity of $\rho_{y x}^{\mathrm{A}_{\text {int }}}=\sigma_{y x}^{\mathrm{A}_{\text {int }}} \rho_{0}^{2} \approx 0.6 \mu \Omega \mathrm{cm}$, which we plot as a horizontal green line in inset, Fig. $5 c$. We find a remarkable agreement with the measured $\rho_{y x}^{\mathrm{A}} \approx 1 \mu \Omega \mathrm{cm}$. 
a

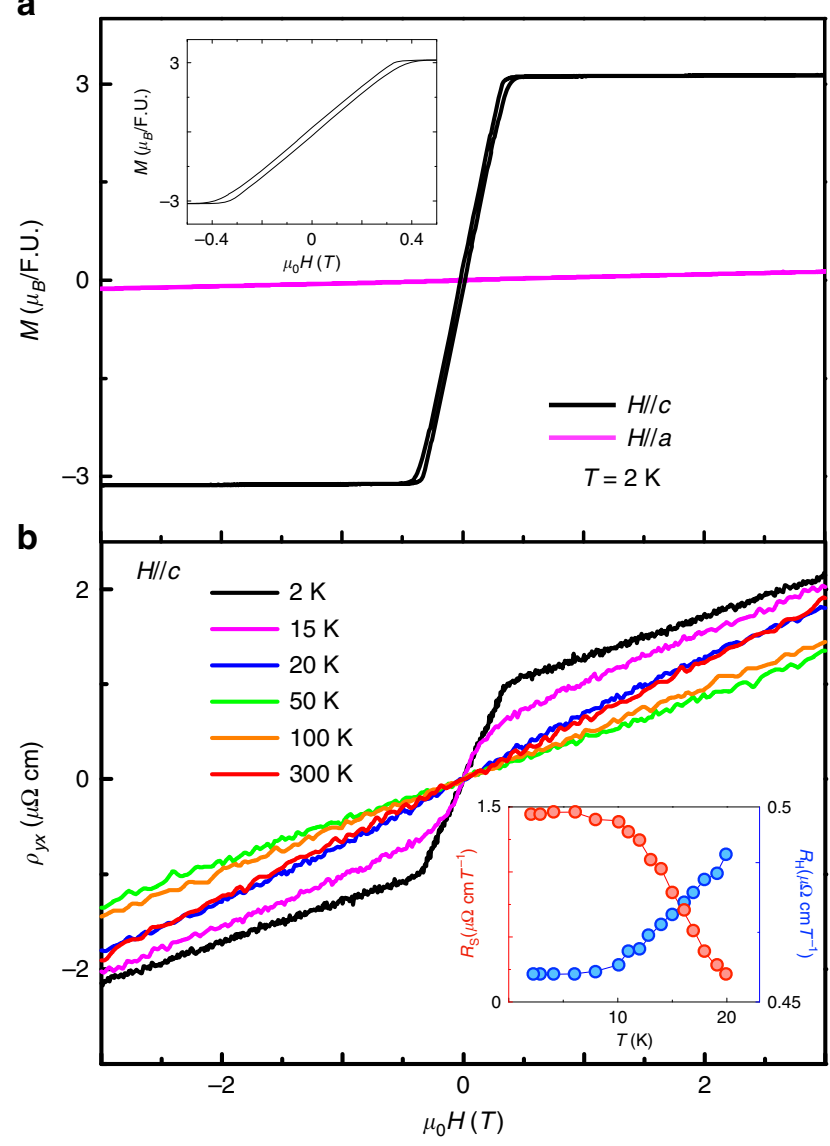

C

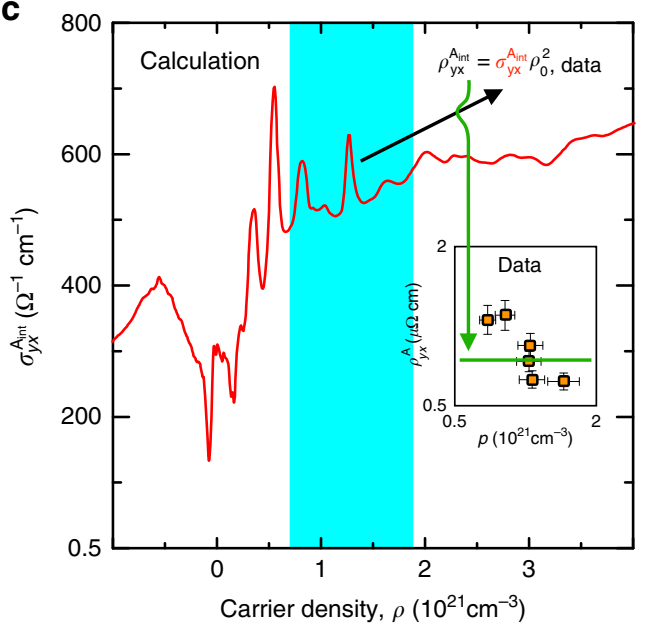

d

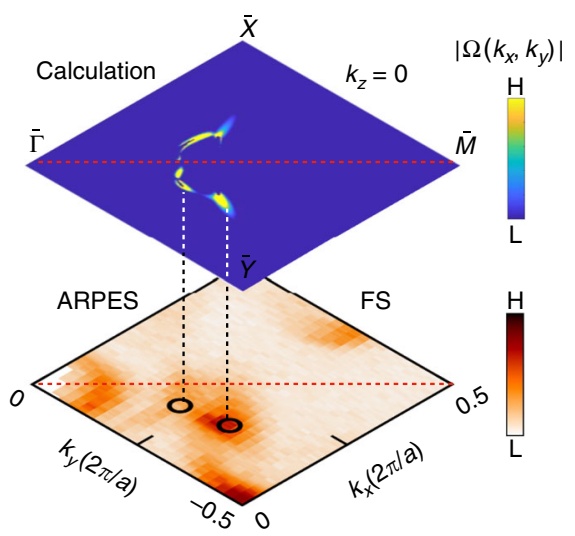

Fig. 5 Observation of intrinsic anomalous Hall transport in PrAlGe. a Magnetization M of PrAlGe along the c-axis (black) and a-axis (magenta) versus magnetic induction $\mu_{0} \mathrm{H}$. The Curie temperature is observed to be $T_{\mathrm{C}}=16 \mathrm{~K}$. Inset: zoom-in showing a hysteresis loop. $\mathbf{b}$ Hall resistivity $\rho_{y x}$ as a function of $\mu_{0} H$. The inset shows the ordinary and anomalous Hall coefficients $R_{\mathrm{H}}$ (blue) and $R_{\mathrm{S}}$ (red) as a function of temperature extracted from the data. c Ab initio calculated intrinsic anomalous Hall conductivity $\sigma_{y x}^{A_{\text {int }}}$ as a function of carrier density. The shaded turquoise area corresponds to the carrier density of the measured PrAlGe samples. The inset shows the measured anomalous Hall resistivity $\rho_{y x}^{\mathrm{A}}$ as a function of carrier density $\mathrm{p}$. The horizontal green line corresponds to the calculated intrinsic contribution to $\rho_{y x}^{\mathrm{A}}$. The error bars are set by the $\pm 10 \%$ error in measurement of the sample dimensions. $\mathbf{d}$ Surfacebulk-transport correspondence of Weyl fermions in PrAlGe. Top: Berry curvature magnitude $|\Omega(k)|$ at $k_{z}=0$ summed over energies below the Fermi level, from ab initio calculation. Bottom: ARPES-measured Fermi surface, suggesting that the Berry curvature field is concentrated near the termination points of the Fermi arc and projection point of the Weyl cones (open circles) observed in ARPES.

This agreement suggests that the Berry curvature dominates the anomalous Hall response in PrAlGe.

Bulk-boundary-transport correspondence in PrAlGe. To study the origin of the Berry curvature fields giving rise to the intrinsic anomalous Hall response, we compare the ARPES-measured Fermi surface with the calculated Berry curvature field. By summing over energies below $E_{\mathrm{F}}$, the Berry curvature magnitude $\mid \Omega$ $(k) \mid$ at $k_{z}=0$ shows concentrated hot spots, see Fig. $5 \mathrm{~d}$. The points of concentrated Berry curvature correspond to the position of the $\mathrm{W}_{3}$ and $\mathrm{W}_{4}$ Weyl fermions. A qualitative comparison with the ARPES-measured Fermi surface shows that within our momentum-space resolution the hot spot region coincides with the termination points of the measured topological Fermi arc. According to ref. ${ }^{36}$, the AHE of an ideal magnetic Weyl semimetal with only one pair of Weyl fermions near the Fermi level can be written as: $\sigma_{\mathrm{Weyl}}=\mathrm{e}^{2} k / 2 \pi h$, where $k$ is their momentum space separation. A quantitative estimate using the measured Weyl fermion separation $k_{\mathrm{Weyl}} \approx 0.15 \AA^{-1}$ yields an intrinsic anomalous hall conductivity $\sigma_{\mathrm{Weyl}} \approx 738 \Omega^{-1} \mathrm{~cm}^{-1}$, consistent with our ab initio calculations. Collectively, our results provide strong evidence suggesting that the measured intrinsic anomalous Hall response arises from the Berry curvature contributions of the Weyl fermions in magnetic non-centrosymmetric $\operatorname{PrAlGe}{ }^{36}$.

\section{Discussion}

The captured surface- and bulk-states in our ARPES spectra and magneto-transport measurements, taken together with support from ab initio calculations, establish a surface-bulk-transport correspondence demonstrating that PrAlGe exhibits novel Berry curvature mediated topological electronic phenomena. Our results open new research directions in understanding and engineering tunable topological electronic properties in the noncentrosymmetric magnet PrAlGe. In particular, due to the absence of both time-reversal and inversion symmetry, exotic types of photogalvanic effects may emerge ${ }^{37-40}$. Additionally, topological currents in PrAlGe may also allow for the development of all-electrical spin generation and injection with no entropy production. Lastly, the soft-ferromagnetism may allow the spin-polarized topological currents to be turned on/off via an external magnetic field ${ }^{41,42}$. The control versatility and potential access to a large number of exotic phenomena makes magnetic 
non-centrosymmetric PrAlGe an exciting material platform for probing topological and quantum matter physics.

\section{Methods}

Single crystal growth. Single crystals of PrAlGe were grown by the self-flux technique with purified Pr ingots (99.9\%), Al shots (99.99\%), and Ge pieces (99.99\%). Stoichiometric mixtures of Pr1Al18Ge1 were set in alumina crucibles and then sealed in fused silica ampoules under partially filled argon atmosphere. After being pegged at $1150{ }^{\circ} \mathrm{C}$ for a few hours, the ampoules were slowly cooled down to the centrifugal temperature $750^{\circ} \mathrm{C}$ at a rate of $0.1{ }^{\circ} \mathrm{C} / \mathrm{min}$.

Angle-resolved photoemission spectroscopy. Low-energy ARPES measurements (VUV-ARPES) were carried out at Beamlines (BL) 5-2 of the Stanford Synchotron Radiation Lightsource (SSRL) at SLAC in Menlo Park, CA, USA, with a Scienta R4000 electron analyzer. The angular resolutions was better than $0.2^{\circ}$, and the energy resolution was better than $20 \mathrm{meV}$. The beam spot size was about $20 \times 40 \mu \mathrm{m}^{2}$. Samples were cleaved in situ and measured under vacuum better than $5 \times 10^{-11}$ Torr and temperatures $<11 \mathrm{~K}$. Soft X-ray ARPES measurements (SX-ARPES) were performed at the ADRESS beamline at the Swiss Light Source in the Paul Scherrer Institut (PSI) in Villigen, Switzerland. The combined (beamline and analyzer) energy resolution of the SX-ARPES measurements varied between 40 and $80 \mathrm{meV}$. The angular resolution of the SXARPES analyzer was better than $0.2^{\circ}$. PrAlGe samples were cleaved in situ under a vacuum condition better than $5 \times 10^{-11}$ Torr and temperature less than the Curie temperature $T_{\mathrm{C}}$.

Magnetization measurements. Magnetization measurements were performed using the Quantum Design Magnetic Property Measurement System (MPMS-3).

Transport measurements. Resistance and Hall effect measurements were performed in a Quantum Design Physical Property Measurement System (PPMS), using the standard four-probe technique with silver paste contacts that were cured at room temperature.

First-principles calculations. The first-principles calculations were performed within the density functional theory (DFT) framework using the projector augmented wave method as implemented in the VASP package ${ }^{43,44}$. The generalized gradient approximation (GGA) was used ${ }^{45}$ for the exchange-correlation effect and the Hubbard energy $U$ used in the calculation is $4 \mathrm{eV}$. A $\Gamma$-centered $k$-point $14 \times$ $14 \times 14$ mesh was used and spin-orbit coupling (SOC) was included in selfconsistent cycles. To generate the (001)-surface states of PrAlGe, Wannier functions were generated using the $d$ and $f$ orbitals of $\operatorname{Pr}$, and the $s$ and $p$ orbitals of $\mathrm{Al}$ and $\mathrm{Ge}$. The surface states were calculated for a semi-infinite slab by the iterative Green's function method. They were optimized based on experimental results ${ }^{46}$.

\section{Data availability}

The data supporting the findings of this study are available within the paper, and other findings of this study are available from the corresponding author upon reasonable request.

Received: 22 February 2019; Accepted: 19 March 2020;

Published online: 03 July 2020

\section{References}

1. Anderson, P. \& Blount, E. Symmetry considerations on martensitic transformations: "ferroelectric" metals? Phys. Rev. Lett. 14, 217 (1965).

2. Belopolski, I. et al. Discovery of topological Weyl fermion lines and drumhead surface states in a room temperature magnet. Science 365,1278 1281 (2019).

3. Chang, G. et al. Topological Hopf and Chain Link Semimetal States and Their Application to $\mathrm{Co}_{2} \mathrm{MnG}$. Phys. Rev. Lett. 119, 156401 (2017).

4. Belopolski, I. et al. Signatures of a topological Weyl loop in $\mathrm{Co}_{3} \mathrm{Sn}_{2} \mathrm{~S}_{2}$. Preprint at https://arxiv.org/abs/2005.02400 (2020).

5. Volovik, G. E. The Universe in a Helium Droplet. (Clarendon, Oxford, 2003).

6. Hasan, M. Z., Xu, S.-Y. \& Bian, G. Topological insulators, topological superconductors and Weyl fermion semimetals: discoveries, perspectives and outlooks. Phys. Scr. T164, 014001 (2015)

7. Hasan, M. Z. et al. Discovery of Weyl fermion semimetals and topological fermi arc states. Ann. Rev. Cond. Mat. Phys. 8, 289-309 (2017).

8. Armitage, N. P., Mele, E. J. \& Vishwanath, A. Weyl and Dirac semimetals in three-dimensional solids. Rev. Mod. Phys. 8, 015001 (2018).

9. Burkov, A. A. Weyl Metals. Ann. Rev. Cond. Mat. Phys. 9, 359-378 (2018).
10. Murakami, S. Phase transition between the quantum spin hall and insulator phases in 3D: emergence of a topological gapless phase. N. J. Phys. 9, 356 (2007).

11. Wan, X. et al. Topological semimetal and Fermi-arc surface states in the electronic structure of pyrochlore iridates. Phys. Rev. B 83, 205101 (2011).

12. Burkov, A. A. \& Balents, L. Weyl semimetal in a topological insulator multilayer. Phys. Rev. Lett. 107, 127205 (2011).

13. $\mathrm{Xu}, \mathrm{G}$. et al. Chern semimetal and the quantized anomalous Hall effect and $\mathrm{HgCr}_{2} \mathrm{Se}_{4}$. Phys. Rev. Lett. 107, 186806 (2011).

14. Chang, G. et al. Magnetic and noncentrosymmetric Weyl fermion semimetals in the RAlGe family of compounds ( $\mathrm{R}=$ rare earth). Phys. Rev. B 97, 041104(R) (2018).

15. Wang, J., Lian, B., \& Zhang, S.-C. Generation of Spin Currents by Magnetic Field in $\mathcal{T}$ - and $\mathcal{P}$-Broken Materials. SPIN 9, 1940013 (2019).

16. Xiao, D., Chang, M.-C. \& Niu, Q. Berry phase effects on electronic properties Rev. Mod. Phys. 82, 1959 (2010).

17. Chang, G. et al. Topological quantum properties of chiral crystals. Nat. Mater. 17, 978-985 (2018).

18. $\mathrm{Wu}, \mathrm{L}$. et al. Giant anisotropic nonlinear optical response in transition metal monopnictide Weyl semimetals. Nat. Phys. 13, 350-355 (2017).

19. Zhong, S., Orenstein, J. \& Moore, J. E. Optical gyrotropy from axion electrodynamics in momentum space. Phys. Rev. Lett. 87, 117403 (2015).

20. Sodemann, I. \& Fu, L. Quantum nonlinear Hall effect induced by Berry curvature dipole in time-reversal invariant materials. Phys. Rev. Lett. 115, 216806 (2015).

21. Deyo, E. et al. Semiclassical theory of the photogalvanic effect in noncentrosymmetric systems. Preprint at https://arxiv.org/abs/0904.1917 (2009).

22. Xu, S.-Y. et al. Discovery of a Weyl Fermion semimetal and topological Fermi arcs. Science 349, 613-617 (2015).

23. Lv, B. Q. et al. Experimental discovery of Weyl semimetal TaAs. Phys. Rev. X 5, 031013 (2015).

24. Soluyanov, A. A. et al. Type-II Weyl semimetals. Nature 527, 495-498 (2015)

25. Xu, S.-Y. et al. Discovery of Lorentz-violating type II Weyl fermions in LaAlGe. Sci. Adv. 3, e1603266 (2017)

26. Son, D. T. \& Spivak, B. Z. Chiral anomaly and classical negative magnetoresistance of Weyl metals. Phys. Rev. B 88, 104412 (2013).

27. Yang, H. et al. Topological Weyl semimetals in the chiral antiferromagnetic materials $\mathrm{Mn}_{3} \mathrm{Ge}$ and $\mathrm{Mn}_{3}$ Sn. N. J. Phys. 19, 015008 (2017).

28. Kuroda, K. et al. Evidence for magnetic Weyl fermions in a correlated metal Nat. Mater. 16, 1090-1095 (2017).

29. Liu, E. et al. Giant anomalous Hall effect in a ferromagnetic kagome-lattice semimetal. Nat. Phys. 14, 1125-1131 (2018).

30. $\mathrm{Xu}, \mathrm{Q}$. et al. Topological surface Fermi arcs in the magnetic Weyl semimetal $\mathrm{Co}_{3} \mathrm{Sn}_{2} \mathrm{~S}_{2}$. Phys. Rev. B 97, 235416 (2018).

31. Wang, Q. et al. Large intrinsic anomalous Hall effect in half-metallic ferromagnet $\mathrm{Co}_{3} \mathrm{Sn}_{2} \mathrm{~S}_{2}$ with magnetic Weyl fermions. Nat. Commun. 9, 3681 (2018).

32. Belopolski, I. et al. Criteria for directly detecting topological Fermi arcs in Weyl semimetals. Phys. Rev. Lett. 116, 066802 (2016).

33. Nagaosa, N. et al. Anomalous Hall effect. Rev. Mod. Phys. 82, 1539-1592 (2010).

34. Coleman, P. et al. Theory for the anomalous Hall constant of mixed-valence systems. Phys. Rev. Lett. 55, 144 (1985).

35. Fert, A. et al. Theory of the Hall effect in heavy-fermion compounds. Phys. Rev. B 36, 1907 (1987).

36. Burkov, A. A. Anomalous Hall Effect in Weyl Metals. Phys. Rev. Lett. 113, 187202 (2014).

37. Chan, C. et al. Photocurrents in Weyl semimetals. Phys. Rev. B 95, 041104 (2017).

38. Ma, Q. et al. Direct optical detection of Weyl fermion chirality in a topological semimetal. Nat. Phys. 13, 842-847 (2017)

39. de Juan, F. et al. Quantized circular photogalvanic effect in Weyl semimetals Nat. Commun. 8, 15995 (2017).

40. Chang, G. et al. Unconventional Photocurrents from Surface Fermi Arcs in Topological Chiral Semimetals. Phys. Rev. Lett. 124, 166404 (2020).

41. Yin, J. et al. Giant and anisotropic electronic response to vectorial magnetizations in a topological magnet. Nature 562, 91-95 (2018).

42. Suzuki, T. et al. Singular angular magnetoresistance in a magnetic nodal semimetal. Science 365, 377-381 (2019).

43. Kresse, G. \& Joubert, D. From ultrasoft pseudopotentials to the projector augmented-wave method. Phys. Rev. B 59, 1758 (1999).

44. Kresse, G. \& Furthmüller, J. Efficiency of ab-initio total energy calculations for metals and semiconductors using a plane-wave basis set. Comput. Mater. Sci 6, 15-50 (1996).

45. Perdew, J. P. et al. Generalized gradient approximation made simple. Phys. Rev. Lett. 77, 3865 (1996)

46. M. Z. Hasan in Conference Proceeding of APS: http://meetings.aps.org/ Meeting/MAR19/Session/B04.3. 
47. Strocov, V. N. et al. High-resolution soft X-ray beamline ADRESS at the Swiss Light Source for resonant inelastic X-ray scattering and angle-resolved photoelectron spectroscopies. J. Synchrotron Rad. 17, 631-643 (2010).

48. Strocov, V. N. et al. Soft-X-ray ARPES facility at the ADRESS beamline of the SLS: concepts, technical realisation and scientific applications. J. Synchrotron Rad. 21, 32-44 (2014).

\section{Acknowledgements}

Work at Princeton and Princeton-led ARPES measurements and theoretical work reported here was supported by the US Department of Energy under a Basic Energy Sciences grant (number DOE/BES DE-FG-02-05ER46200). This research used resources of the Stanford Synchrotron Radiation Lightsource (SSRL), which is a DOE Office of Science Facility, under contract number DE-AC02-76SF00515. S.J. was supported by the National Natural Science Foundation of China (U1832214, 11774007), the National Key R\&D Program of China (2018YFA0305601) and the Key Research Program of the Chinese Academy of Science (grant number XDPB08-1). J.M. was supported by the National Natural Science Foundation of China (11774223, U1732154) and a Shanghai talent program. H.L. acknowledges the support by the Ministry of Science and Technology (MOST) in Taiwan under grant number MOST 109-2112-M-001-014-MY3. T.A.C. was supported by the National Science Foundation Graduate Research Fellowship Program under Grant No. DGE-1656466. M.Z.H. acknowledges support from the Miller Institute of Basic Research in Science at the University of California at Berkeley and Lawrence Berkeley National Laboratory in the form of a Visiting Miller Professorship during the early stages of this work. M.Z.H. also acknowledges visiting scientist support from IQIM at the California Institute of Technology. We thank D. Lu and M. Hashimoto for beamline support at SSRL in Menlo Park, California. We also thank D. Multer for his assistance in the soft X-ray ARPES measurements. The soft X-ray photoemission data presented in Fig. 4 was collected at the ADRESS beamline of the Swiss Light Source ${ }^{47,48}$. The authors acknowledge synchrotron radiation beamtime at the ADRESS beamline of the Swiss Light Source of the Paul Scherrer Institut in Villigen, Switzerland under Proposal 20170898. Specifically, we acknowledge the important contributions of Vladimir N. Strocov, Alla Chikina, and Niels B. M. Schröter in the acquisition of the soft $\mathrm{X}$-ray ARPES spectra.

\section{Author contributions}

M.Z.H., D.S.S., and G.C. conceived the project.; D.S.S., I.B., T.A.C., and N.A. conducted the VUV-ARPES measurements in consultation with S-Y.X. and M.Z.H.; I.B. carried out the soft X-ray ARPES measurements in consultation with D.S.S., S-Y.X., N.A., and M.Z.H.; H.L. and S.J. conducted the magneto-transport measurements. X.X., X.Z., Y.B.,
Y-Y.L., and S.J. synthesized the PrAlGe samples; J.M. performed the single crystalline X-ray diffraction measurements. G.C. and H.L. performed the first-principles/density functional theory calculations; D.S.S., G.C., and I.B. performed the analysis, interpretation and figure development in consultation with J-X.Y., G.B. N.A., S.S.Z., S-Y.X. and M.Z.H.; D.S.S. wrote the manuscript in consultation with G.C. I.B., J-X.Y., G.B., S-Y.X. and M.Z.H.; M.Z.H. supervised the project.

\section{Competing interests}

The authors declare no competing interests.

\section{Additional information}

Supplementary information is available for this paper at https://doi.org/10.1038/s41467020-16879-1.

Correspondence and requests for materials should be addressed to M.Z.H.

Peer review information Nature Communications thanks the anonymous reviewers for their contribution to the peer review of this work.

Reprints and permission information is available at http://www.nature.com/reprints

Publisher's note Springer Nature remains neutral with regard to jurisdictional claims in published maps and institutional affiliations.

(c) Open Access This article is licensed under a Creative Commons Attribution 4.0 International License, which permits use, sharing, adaptation, distribution and reproduction in any medium or format, as long as you give appropriate credit to the original author(s) and the source, provide a link to the Creative Commons license, and indicate if changes were made. The images or other third party material in this article are included in the article's Creative Commons license, unless indicated otherwise in a credit line to the material. If material is not included in the article's Creative Commons license and your intended use is not permitted by statutory regulation or exceeds the permitted use, you will need to obtain permission directly from the copyright holder. To view a copy of this license, visit http://creativecommons.org/ licenses/by/4.0/.

(c) The Author(s) 2020, corrected publication 2022 